\section{Comment on: Vascular cell adhesion molecule-1 in rheumatoid arthritis}

\section{To the Editor}

Salem and Zahran ${ }^{1}$ conducted a cross sectional study investigating the role of VCAM-1 in patients with rheumatoid arthritis (RA), its relation to disease activity, oxidative stress and systemic inflammation. ${ }^{1}$ They studied 50 patients with early newly diagnosed RA (less than 6 month duration), 50 patients with chronic disease and 50 healthy, aged matched controls. They found that patients with RA showed significantly higher serum vascular cell adhesion molecule-1 (VCAM-1), malondialdehyde (a marker of oxidative stress), disease activity index 28 (DAS-28), erythrocyte sedimentation rate (ESR), C-reactive protein, triglycerides, total cholesterol, low-density lipoprotein (LDL), and atherogenic index levels than the control group. Rheumatoid arthritis patients, more so the chronic cases, had significantly lower total antioxidant capacity and high-density lipoprotein (HDL) levels than control group. There was significant positive correlation between serum VCAM-1, DAS-28, disease activity markers (CRP and ESR) and oxidative stress with newly diagnosed patients with RA had significantly had higher levels of disease activity, lipid profile, atherogenic index, oxidative stress and serum VCAM-1 than patients with chronic disease. Disease activity, oxidative stress and VCAM-1 level were lower in treated patients.

Diseases, characterized by chronicinflammation, such as RA carry a higher than normal risk for cardiovascular and cerebrovascular disease, probably as a result of the increased prevalence of the usual risk factors as well as the chronic inflammatory burden. ${ }^{2}$ Rheumatoid arthritis is a chronic systemic immune mediated disease, characterized by symmetrical polyarthritis especially the small joints in the hands. Often, the synovial inflammation is accompanied by inflammation in the in the wall of blood vessels. There are similarities in the abnormal adaptive immune responses seen in both RA and atherosclerosis. In addition, patients with active $\mathrm{RA}$, are in a hypercoagulable state due to slow mobility and increased plasma levels of acute phase reactants such as fibrinogen and von Willebrand factor. Drugs such as NSAIDS and long term corticosteroid use may also contribute to the development of cardiovascular events [Figure 1].

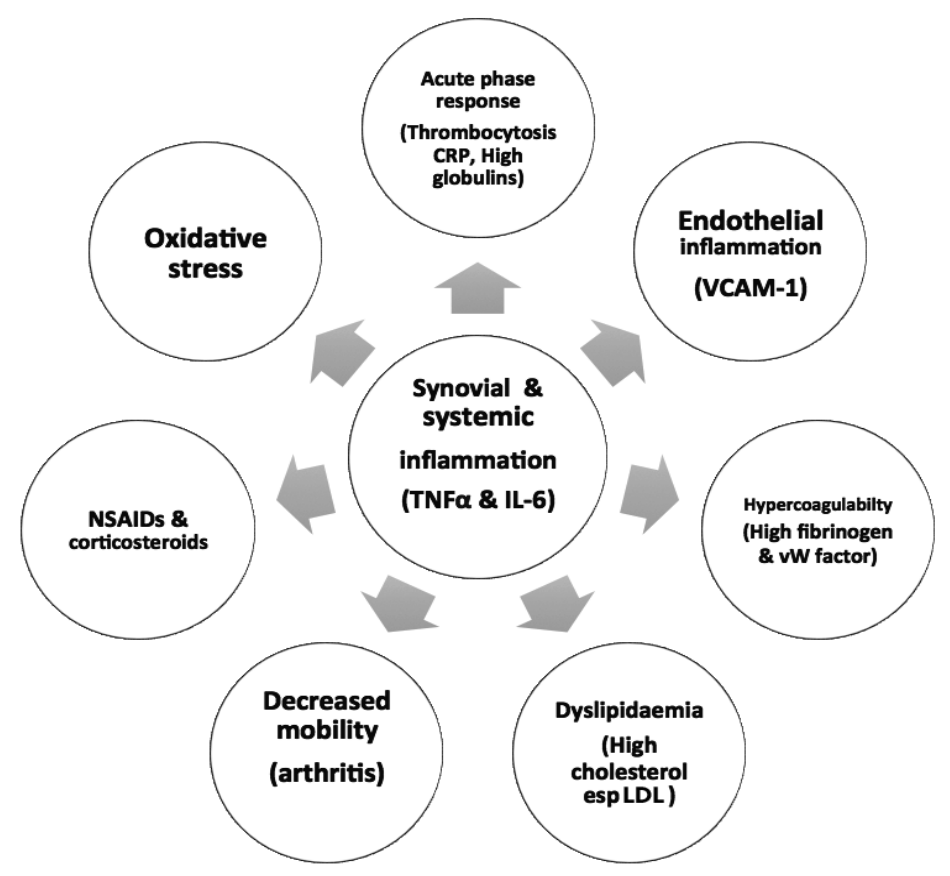

Figure 1 - Contributing factors for increased cardiovascular events in active rheumatoid arthritis. CRP: C-reactive protein, VCAM1: vascular cell adhesion molecule-1, NSAID: nonsteroidal anti-inflammatory drugs, TNF- $\alpha$ : tumor necrosis factor- $\alpha$, IL-6: Interleukin 6, LDL: low density lipoprotein, vW factor: von Willebrand factor 
The synovial inflammation is predominantly mediated by tumor necrosis factor-alpha (TNF- $\alpha$ ) in most cases of RA. TNF- $\alpha$, a member of the TNF ligand superfamily, is mainly produced by macrophages, $\mathrm{T}$ lymphocytes and natural killer cells. The soluble form of TNF- $\alpha$ binds to TNF receptor 1 (TNFR1), a type I transmembrane protein, which is expressed in almost all cells. This binding leads to inflammation, apoptosis, reactive oxygen species generation and cell proliferation. Tumor necrosis factor-alpha receptor 1 induces several intracellular signalling pathways including intracellular adhesion molecule-1 (ICAM-1), and vascular cell adhesion molecule-1 (VCAM-1). Vascular cell adhesion molecule-1 is expressed in endothelial cells and in severe inflammation, it is also expressed on the surface of other cells, including tissue macrophages, dendritic cells, bone marrow fibroblasts, myoblasts and others. Vascular cell adhesion molecule-1 mediates leucocyte endothelial cell adhesion and signal transduction. It has been shown to play a role in the pathogenesis of both RA and atherosclerosis. ${ }^{3}$

The findings of this study lend support to the importance of treating patients with RA as early as possible, using standard and targeted therapies with the aim of effectively controlling the systemic inflammation and achieving complete or near remission. This will lead to improvement in functional ability of patients, lowering oxidative stress, normalization of lipid dysfunction and lowering of serum VCAM-1. Achieving remission or at least decreasing disease activity will improve the long term outcome in RA and lower the incidence of comorbidities such as cardiovascular disease. There is good evidence from a recent study from Denmark showing a narrowing of the mortality gap for patients with RA and the general population but the rate of cardiovascular disease remains high among RA patients. ${ }^{2}$ Though there has been significant advances in targeted therapy for patients with RA over the last 20 years, rheumatologists need to do more for their patients with RA aiming at achieving remission or near remission to reduce the development of comorbidities which are often the cause of death in RA.
Saudi Med J 2021; Vol. 42 (8): 918-920 doi: 10.15537/smj.2021.42.8.918

\section{Reply from the Author}

No reply was received from the Author.

\section{References}

1. Salem, HR, Zahran ES. Vascular cell adhesion molecule-1 in rheumatoid arthritis patients: Relation to disease activity, oxidative stress, and systemic inflammation. Saudi Med J 2021; 42: 620-628.

2. Løgstrup BB, Ellingsen T, Pedersen AB, et al. Cardiovascular risk and mortality in rheumatoid arthritis compared with diabetes mellitus and the general population. Rheumatol 2021; 60:1400-1409.

3. Kong DH, Kim YK, Kim MR, Jang JH, Lee S. Emerging Roles of Vascular Cell Adhesion Molecule-1 (VCAM-1) in Immunological Disorders and Cancer. Int J Mol Sci 2018; 19: 1057. 
Dear Author, please read through your article carefully, make a critical review and highlight any changes you require to be made, please also list these clearly on a separate sheet.

If you don't have any more query/correction, kindly sign on every page of the paper and send it back to us with a covering letter stating that you approve the publication of your paper.

After you have signed and returned the signed proof of your article, the Journal will not be held responsible for any errors that appear in the final print.

Many thanks. 